\title{
Atlantic Equatorial Deep Jets: Space-Time Structure and Cross-Equatorial Fluxes
}

\author{
Uwe Send, Carsten Eden, * AND Friedrich Schott \\ Institute für Meereskunde, Kiel, Germany
}

(Manuscript received 6 August 1999, in final form 12 July 2001)

\begin{abstract}
The so-called equatorial stacked jets are analyzed with ship-board observations and moored time series from the Atlantic Ocean. The features are identified and isolated by comparing vertical wavenumber spectra at the equator with those a few degrees from the equator. Mode-filtering gives clear views of the jets in meridional sections, the typical extent being $\pm 1^{\circ}$ in latitude. The vertical structure can be well described (explaining $82 \%$ of the variance) by $N^{-1}$-stretched cosines, with a Gaussian amplitude tapering in the vertical. The stretched wavelengths are somewhat variable.

Fitting jets of a fixed (stretched) wavelength to four moored sensors in the depth range 1300-1900 m, allows one to track the vertical phase of the jets with an rms error of $30^{\circ}-45^{\circ}$. The resulting fit from a 20 -month moored time series shows long periods of unchanging jet conditions and intermittent times of high variability. There is no significant vertical propagation on these timescales nor a seasonal reversal. Using a composite from many different experiments, interannual variability is visible, however.

A possible mechanism for the stacked jets is inertial instability, resulting from background meridional shears at the equator. A condition is that the Ertel potential vorticity becomes zero somewhere, due to meridional asymmetries in the zonal flows. The ship-board observations show that this may be approximately fulfilled by the instantaneous zonal low-mode flows at various depths, resulting from an excess of zonal momentum south of the equator most of the time. Inertial instability should act to redistribute this zonal momentum, and our mooring data show indeed persistent northward momentum flux, but not at the depth levels expected. The momentum transport might suggest that the jets can also flux or mix other properties across the equator.
\end{abstract}

\section{Introduction}

In the last three decades, observations have revealed alternating eastward and westward currents located below the thermocline at the equator throughout the three ocean basins. Lutyen and Swallow (1976) first described these so-called deep jets in the Indian Ocean, Hayes and Milburn (1980) and Leetma and Spain (1981) observed these features in the Pacific. The first hint for the existence of the deep jets in the Atlantic Ocean was given by Eriksen (1982) deduced from hydrographic data and later from Ponte and Luyten (1990) based on a single profile of direct current measurements.

These observations characterized the deep jets as strong zonal flows with amplitudes of up to $0.25 \mathrm{~m} \mathrm{~s}^{-1}$, found from 500- to 3000-m depth, trapped within about one degree at the equator, and with typical vertical scales of 100-300 m. The meridional and vertical structure of the jets in the Pacific Ocean is fairly well defined, due

\footnotetext{
* Current affiliation: Dalhousie University, Halifax, Nova Scotia, Canada.
}

Corresponding author address: Uwe Send, Duesternbrooker Weg 20, 24105 Kiel, Germany.

E-mail: usend@ifm.uni-kiel.de to extensive observations from the PEQUOD Experiment in 1981-83 (Firing 1987). The zonal and temporal scales of the jets are less well observed, but Firing (1987) and Ponte and Luyten (1989) found that the deep jets in the Pacific Ocean lacked significant vertical propagation on timescales of $1 \mathrm{yr}$ or longer and had a zonal extent of at least $10^{\circ}$ of longitude.

For the Atlantic Ocean, data from German and French equatorial cruises in the past years are now allowing a more detailed characterization of the deep jet features there. Gouriou et al. (1999) used ship-board current profiles from three such cruises and a historical profile to document the vertical scales and amplitudes of the jets in the Atlantic, giving $400-600 \mathrm{~m}$ and $10-20 \mathrm{~cm} \mathrm{~s}^{-1}$, respectively.

Thus, qualitatively the vertical structure of the jets appears to be similar in all three oceans, with somewhat larger scales in the Atlantic, but a quantitative or analytical description is still lacking. The meridional extent is $1^{\circ}-1.5^{\circ}$ in all oceans. There remain some questions about how well the jets are centered on the equator and whether some of them are offset toward the so-called dynamical equator (Hua et al. 1996). The state of knowledge about the zonal structure and especially about the temporal behavior is generally still unsatisfactory.

Several attempts have been made to interpret these 


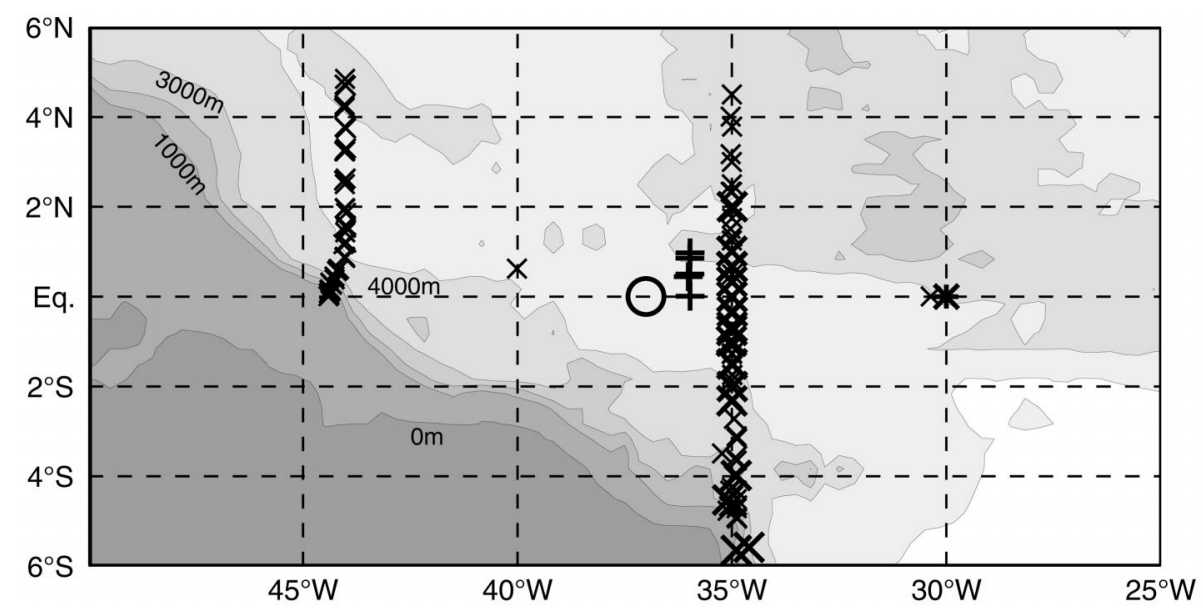

FIG. 1. The location of the shipboard observations (crosses) and the mooring (large circle) in the tropical Atlantic. The big crosses denote locations of the three french cruises, the plus-signs denote the NOAA-AOML cruise, the single big star the published profile of Ponte et al. (1990), and the small crosses the four German Meteor cruises.

features as linear equatorial waves. Wunsch (1977) suggested that the deep jets in the Indian Ocean might be vertically propagating equatorially stacked waves forced by the monsoonal wind system. McCreary (1984) found jetlike structures in his model, where time-dependent zonal wind forcing creates a complex response of Kelvin and Rossby waves, but the period of the waves was at most $1 \mathrm{yr}$ in contradiction with the above described observations. McCreary and Lukas (1986) modeled stationary Kelvin waves, forced by a wind patch and an interaction with a mean barotropic current, with an amplitude of $15 \mathrm{~cm} \mathrm{~s}^{-1}$. Muench et al. (1994) found that the deep jets perturb potential vorticity, inconsistent with the hypothesis that the jets are Kelvin waves. They found that the structure of the observed jets resembles that of a long Rossby wave, but the meridional scale was not in accordance with the observations.

Another attempt to model these jets has been via inertial instability theory, put forward by Hua et al. (1996, hereafter H96). There, an adverse (unstable) distribution of absolute angular momentum around the equator leads to an instability in the form of a system of vertically stacked zonal currents with high vertical wavenumber (Dunkerton 1981, 1983; Stevens 1983). The observational support for this process however was very sparse in H96, and the necessary criterion (zero Ertel potential vorticity) was only found to be approached to some extent within the jets of the correct sign and not in the larger-scale background flow.

In the present paper, the spatial and temporal behavior of the deep jets in the Atlantic Ocean is analyzed, using current profiles from eight cruises from 1989 to 1996 [including those of Gouriou et al. (1999)] plus a 20month moored time series of currents at four levels in the regime of the jets. These data have allowed a more complete description of the vertical structure and temporal evolution of the features, and at the same time enable us to revisit the conditions for inertial instability theory.

\section{Database and identification of the jets}

The direct ship-board current observations originate from four German Meteor cruises in the tropical Atlantic (M14, M16, M22, M27) in October 1990, May 1991, November 1992, and March 1994, from three French cruises (Cither 1, Romanche 3, and Etambot 2) in January-February 1993, October-November 1994, and April-May 1996, from one National Oceanic and Atmospheric Administration-Atlantic Oceanographic and Meteorological Laboratory (AOML) cruise in June 93, and a published profile (Ponte and Luyten 1990) from January 1989.

The flow profiles were collected with either the Pegasus profiling system (Spain et al. 1981) or the lowered ADCP method (Fischer and Visbeck 1993), mostly over short meridional sections across the equator near $35^{\circ} \mathrm{W}$ (see the map in Fig. 1). One section in the eastern equatorial Atlantic (near $12^{\circ} \mathrm{W}$, in the vicinity of the Romanche Fracture zone) was not used here since our analyses indicate a different dynamical regime. The other sources of information are time series from four current meters on an equatorial mooring at $36^{\circ} \mathrm{W}$ covering the period of October 1992 through May 1994, thus overlapping with four of the ship-board surveys. The mooring location is also shown in Fig. 1.

The deep jets are embedded in background flows of similar or larger amplitudes, and for our subsequent analyses it is important to be able to separate the jet features from this lower wavenumber background. In order to define the jets, we first compared the vertical wavenumber energy in equatorial zonal current profiles to those profiles away from the equator. To apply conventional spectral analysis methods, the profiles are 
a)

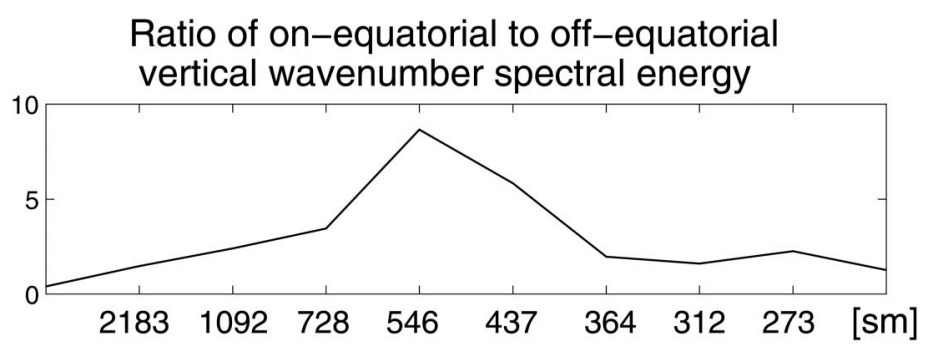

b)

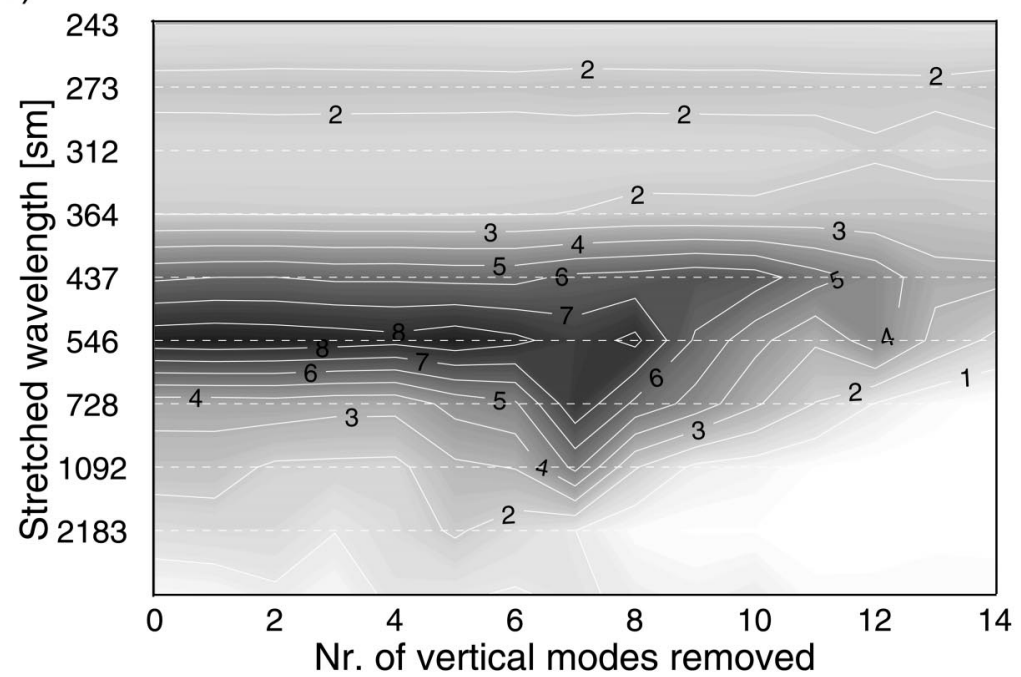

FIG. 2. The ratio of on-equatorial to off-equatorial vertical wavenumber spectral energy. The spectra were computed from $N$-stretched profiles of zonal velocity (54 profiles from six cruises) at the equator and more than $1.5^{\circ}$ from the equator. (a) Distribution of the energy ratio as a function of stretched wavelength. In the range of 400-700 m (referenced to $1500 \mathrm{~m}$ ) there is a maximum of equatorial energy enhancement that represents the unique equatorial features at these wavelengths, i.e., the stacked jets. (b) The energy ratio contoured as a function of stretched wavelength and of the number of removed lowwavenumber linear modes.

stretched and scaled using a WKBJ approximation (Ponte and Luyten 1989) to give a transformed vertical coordinate and corresponding velocities, using a mean buoyancy frequency profile computed from the CTD data. The reference frequency was chosen as the buoyancy frequency at $1500 \mathrm{~m}$, so that the vertical wavelength equals the stretched vertical wavelength at that depth. Relative to unstretched vertical modes, the stretched harmonics are expanded above $1500 \mathrm{~m}$ and compressed below.

The estimated ratio of on-equatorial to off-equatorial energy reaches a maximum at stretched wavelengths of 400-700 m, showing that at those vertical scales features exist at the equator with clearly elevated variance (Fig. 2a). Taking this as the definition of the jets, it was then tested to see how many vertical modes could be removed from the flow profiles before the energy maximum at $546 \mathrm{~m}$ starts to drop off. Figure $2 \mathrm{~b}$ shows that this is the case for eight vertical modes. Therefore, mode filtering with eight dynamical modes will be used to separate the deep jets from the background flow. The later results do not depend qualitatively on this choice.

\section{Spatial structure}

The mode filtering defined in the previous section can now be used to enhance the visibility of the jets in individual meridional sections across the equator. The resulting current distributions are shown in Fig. 3, for the cruises where several profiles across the equator were available. The jets are seen to be confined within $1^{\circ}-1.5^{\circ}$ of the equator, as was found also in the other oceans. Their typical depth range here is $1000-2000 \mathrm{~m}$, even though at times they are found from 500 to 2500 $\mathrm{m}$. The maximum amplitude is usually around $1500 \mathrm{~m}$, typical currents are $10 \mathrm{~cm} \mathrm{~s}^{-1}$.

In the zonal direction, the database is not sufficient to construct sections of the flow. Thus, the large zonal coherence also found in the other oceans can be suggested only with the few sections that were carried out 

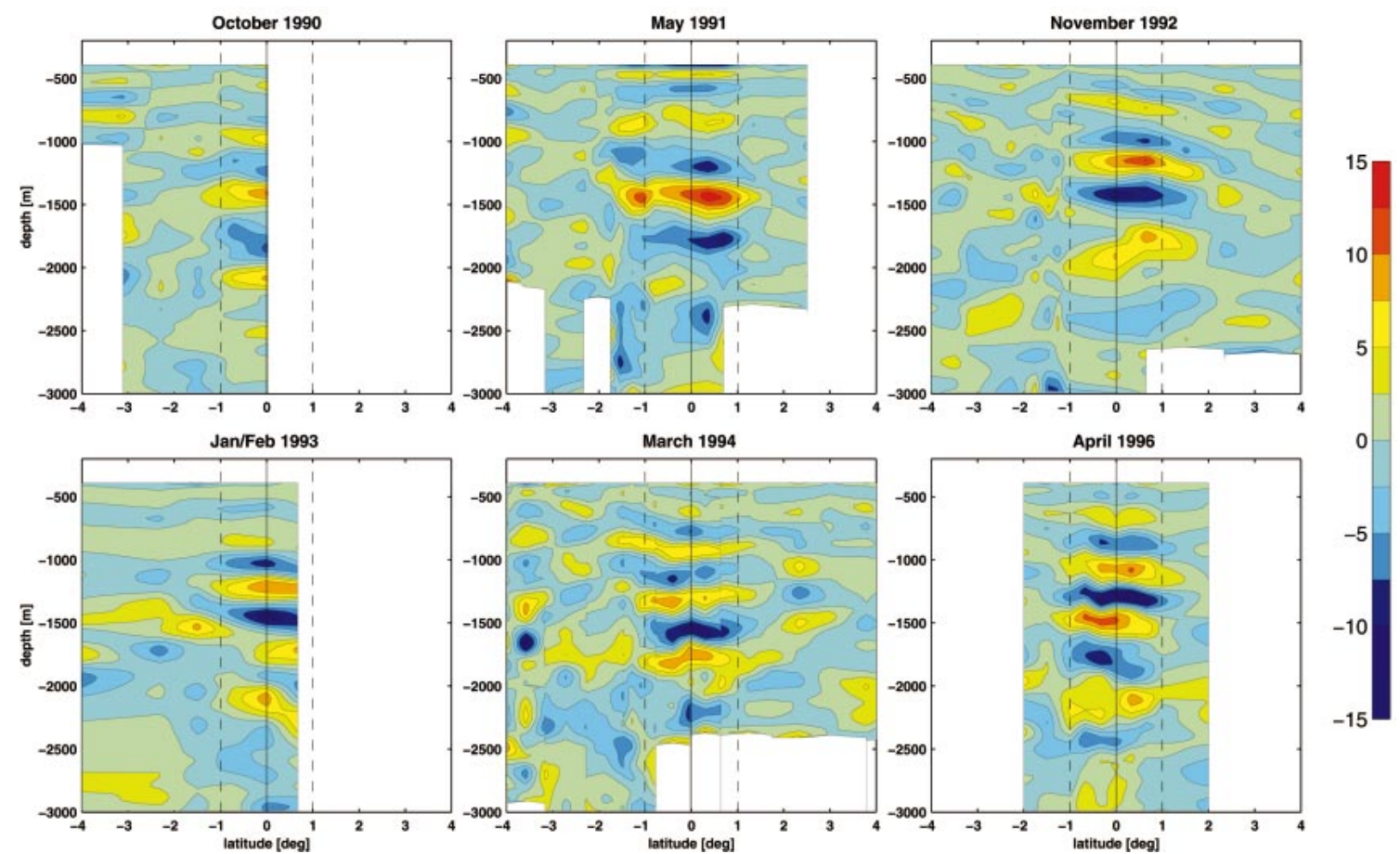

FIG. 3. Meridional sections of zonal flow from six cruises after mode filtering in $\mathrm{cm} \mathrm{s}^{-1}$. The dashed lines are at $\pm 1^{\circ}$ lat, marking the typical meridional extent of the jets.

at $44^{\circ} \mathrm{W}$ together with the observations at $35^{\circ} \mathrm{W}$, plus two individual profiles at other longitudes. In Fig. 4 all the mode-filtered near-equatorial profiles are plotted in an offset manner corresponding to their geographic location. The figure shows that generally the profiles from different longitudes have maxima at similar depths, but that both the shape of the profiles and the exact depth of maxima/minima are not in close agreement. The qualitative result is that there appears to be some degree of zonal coherence over at least $10^{\circ}-15^{\circ}$ in longitude, as also found in previous studies and the other oceans. Note that at $44^{\circ} \mathrm{W}$ one might expect the proximity of the continental slope (oriented NW-SE) to be important, but the jets are still found there and no modified orientation could be detected.

An important issue for the temporal analysis from the moored data in the next section is a more quantitative description of the vertical structure of the jets. Our analyses have shown that the vertical wavelength varies with depth and seems to scale approximately with $N^{-1}$. The $N^{-1}$-stretched wavelengths usually lie in the range of 400-700 m, depending on realization. The vertical variation of the amplitude of the jets however does not obey any traditional $N$-related scaling. Therefore, the observed amplitude variation with its maximum at middepth is described here empirically using a Gaussian envelope centered at $1500 \mathrm{~m}$, with an adjustable depth scale, which was found to be in the range $600-1000 \mathrm{~m}$, depending on realization. The resulting model shape used subsequently for the vertical structure of the jets therefore consists of $N^{-1}$-stretched sines/cosines with variable wavelengths in the 400-700-m range, modulated by a Gaussian envelope with a depth scale of 600 $1000 \mathrm{~m}$.

This model shape was least squares fitted to all available mode-filtered profiles showing jets (37 profiles from eight cruises) over the depth range of 400-3000 $\mathrm{m}$. Overall, $82 \%$ of the variance in the profiles could be explained with this model, leaving a residual misfit of $2 \mathrm{~cm} \mathrm{~s}^{-1} \mathrm{rms}$. The (stretched) wavelengths were mostly in the range $450-650 \mathrm{~m}$. Figure 5 shows eight representative examples (best and worst cases) of how well the depth structure of the jets can be approximated by the model functions, supporting in particular the $\mathrm{N}$ stretched scaling of wavenumber.

\section{Mooring time series and temporal behavior}

The challenge of the analysis presented in this section consists of using only four current meters to estimate the jet features in the presence of an unknown background flow (mode filtering cannot be used with only four points in the vertical). The main property of interest here is the vertical phase of the jet system, in order to address vertical propagation, seasonal reversals, and stationarity. Fortunately the vertical placement of the current meters $(1300,1500,1700,1900 \mathrm{~m})$ was suitable for this analysis (this was not the case for other moorings available at $44^{\circ} \mathrm{W}$ ) since the four depths covered approximately one wavelength of the jets, thus allowing 
May 1991
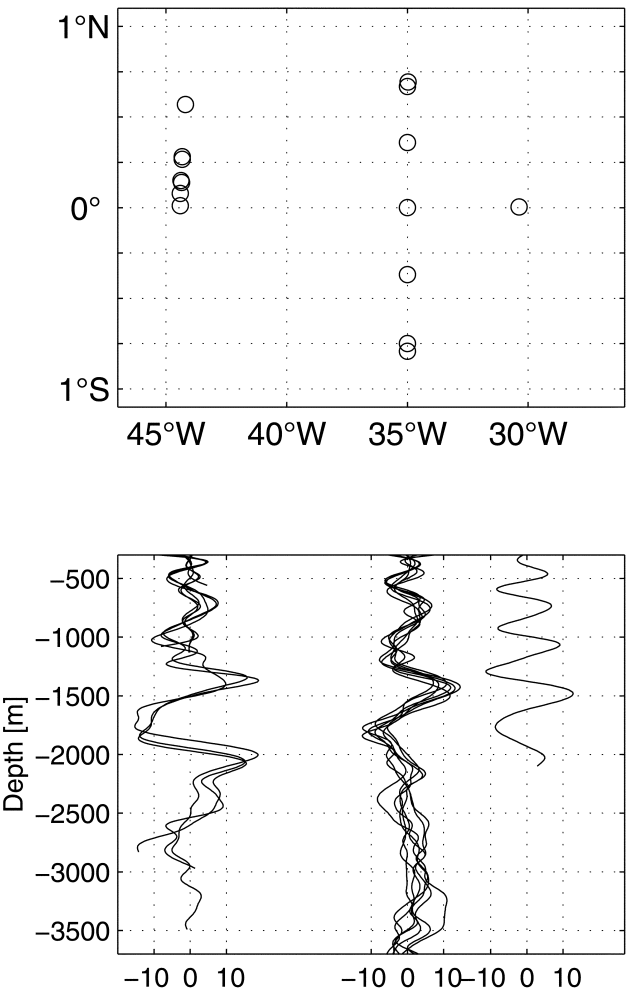

March 1994
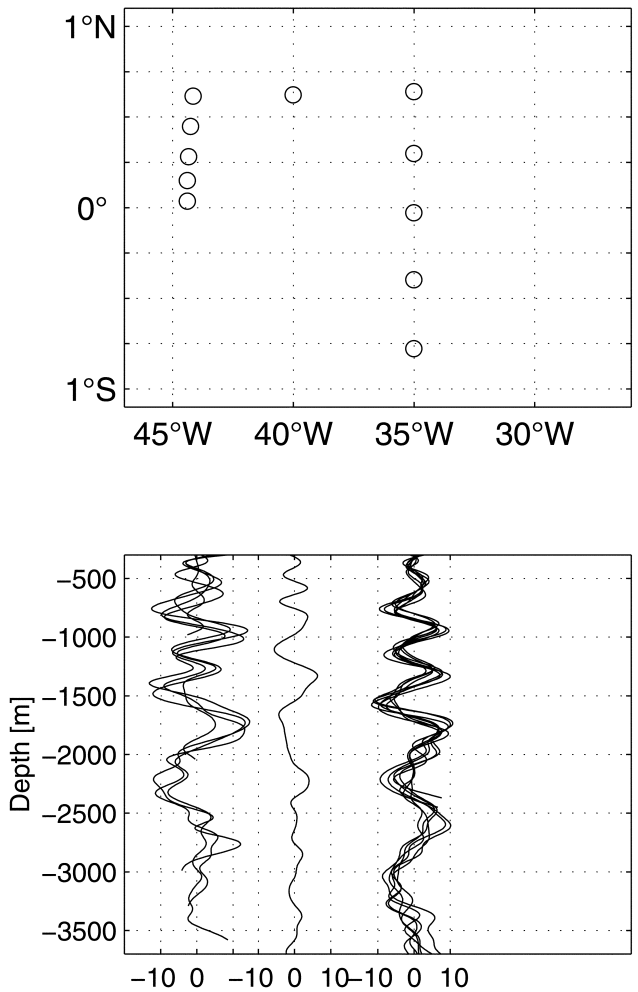

FIG. 4. Examples from two cruises showing the zonal coherence of the jets. (bottom) Mode-filtered profiles of zonal currents within $1^{\circ}$ of the equator are plotted at several zonal locations (bottom). (top) The respective station sites. Our other cruises show similar coherence between the $44^{\circ} \mathrm{W}$ and $35^{\circ} \mathrm{W}$ locations.

a usable determination of the vertical phase of the jets. With the data available, we can determine at most four unknowns describing the vertical profiles to be estimated.

To identify the jets from the mooring data, we therefore use an a priori model, which consists of a stretched sine and cosine with unknown amplitude but fixed wavelengths $(550 \mathrm{~m})$ and a fixed Gaussian amplitude tapering of $800-\mathrm{m}$ vertical scale. The background flow is described by an unknown constant over the depth range of the current meters, so the inversion is formally still overdetermined as in the original model, where we used the full profile data in the depth range of 400$3000 \mathrm{~m}$

The ability of this model to estimate the jets and their vertical phase was tested by simulating the mooring sampling with the full ship-board profiles analyzed in the previous section. Thus, all 37 profiles were sampled at the four current meter depths, the model with fixed wavelength and Gaussian tapering was applied, and the results then compared to the full (mode-filtered) jet profiles available. The rms misfit is larger now $\left(4 \mathrm{~cm} \mathrm{~s}^{-1}\right)$, since the vertical scales/wavelengths are not adjustable anymore and the number of observations sampling the profiles are reduced. The rms error in the vertical phase of the jets is $30^{\circ}$. Figure 6 shows eight examples of these simulations.

For the case that the 37 profiles used are not sufficiently representative (due to many similar profiles), two Monte Carlo simulations were additionally performed. A large number of synthetic jet profiles was generated, with fixed wavelength $(550 \mathrm{~m})$ and envelope scale $(800$ $\mathrm{m}$ ) in the first case, and with variable wavelength and envelope scale (covering the range of observed scales) in the second case. To that, random noise was added in the form of vertical modes, with energy relative to the jets taken from our observations. Then the profiles were sampled again at the four points corresponding to the current meter depths, and the jet parameters were estimated. For the simulation with fixed scales, the rms phase error was $30^{\circ}$, which is an uncertainty in the depth of the jet of $50 \mathrm{~m}$. With variable jet scales, the uncertainty increases to $70 \mathrm{~m}$, which is approximately $45^{\circ}$ at $1500 \mathrm{~m}$.

The thus established technique of fitting the jet structures to the mooring data was then applied to the 20month time series of zonal currents at the four depths given earlier. The result is presented in the upper panel of Fig. 7, which shows a time series of the vertical jet profiles as a contour plot. Periods when the variance of 

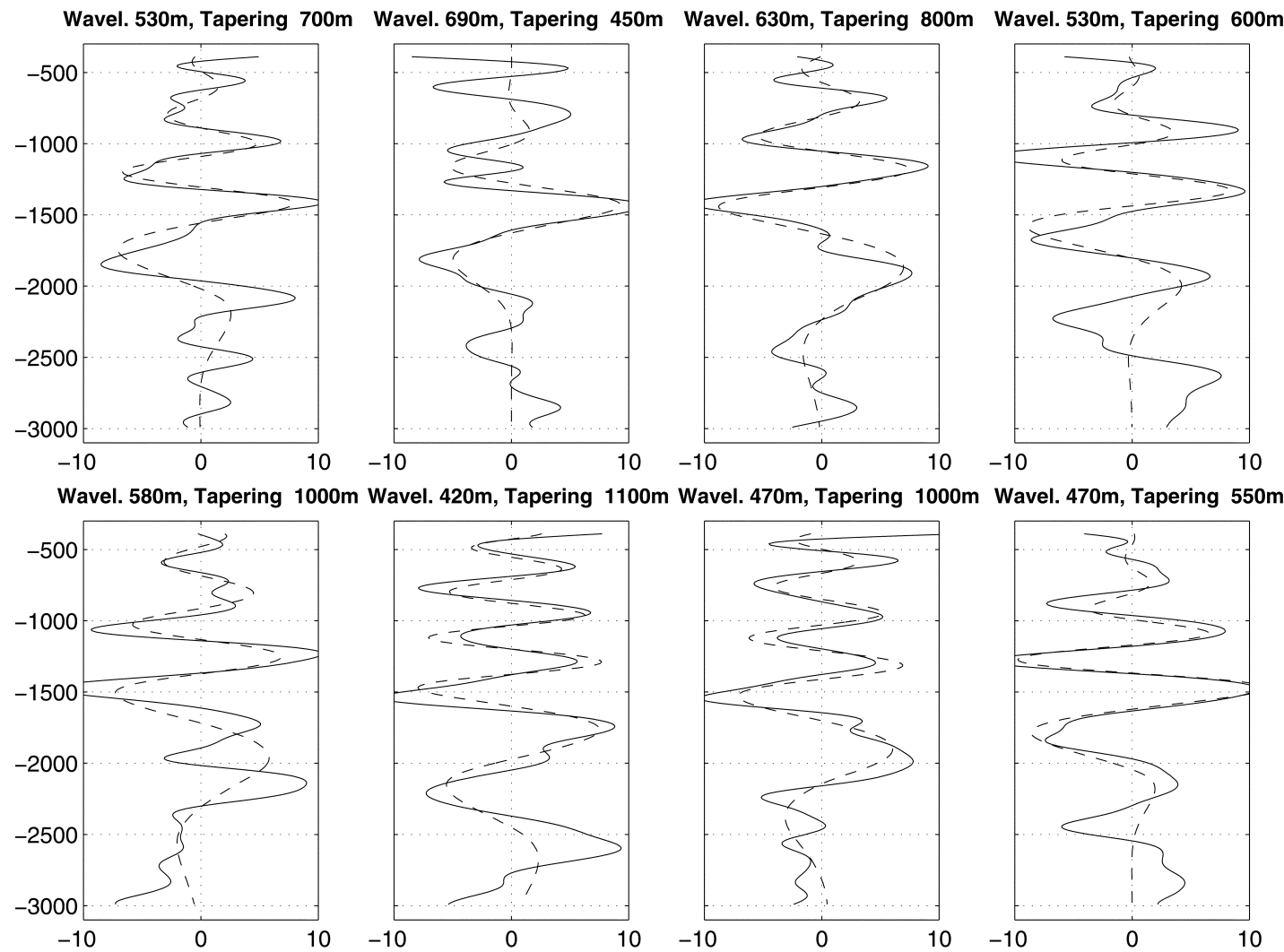

FIG. 5. Some examples of fitting the mode-filtered zonal currents (solid) with $N^{-1}$-stretched sine/cosine functions of variable wavelength (dashed), tapering the amplitude with a Gaussian envelope of variable scale, centered on $1500 \mathrm{~m}$. The optimum wavelengths and depth scales for the envelope are marked above each panel.

all current meters was low are blanked out, since then the approach is expected to be ill-determined. The jets are clearly visible with amplitudes of $10-15 \mathrm{~cm} \mathrm{~s}^{-1}$. It should be pointed out that the purpose of this figure is to document vertical phase behavior and not the amplitude variation. The latter may artificially fluctuate due to varying agreement of the used and actual wavelengths. During this mooring deployment, there are long periods of stationarity, but intervals with intermittent behavior are also visible. The period with the longest stationarity (to within our phase uncertainty) is approximately eight months. Given the phase uncertainty of up to $45^{\circ}$, this suggests that vertical propagation during that period, if it exists, must be slower than 1 cycle in 5 yr. On the other hand, the intermittent changes, seen, for example, in June and October 1993 and February 1994, are significant, since they correspond to more than $90^{\circ}$ phase variation. There is also clearly no seasonal cycle with reversals of the flow, as speculated in previous studies (Gouriou et al. 1999), nor is a vertical propagation visible. The lower panel of Fig. 7 demonstrates that the fitted vertical phase from the mooring data is consistent with shipboard observations to within the estimated phase error, which corresponds to $50-70$ $\mathrm{m}$ rms in vertical position of the maxima/minima.
The time series from the mooring data was not long enough to document complete reversals of the zonal jet currents, as have been reported previously (Gouriou et al. 1999). In order to bring together all temporal information available for the Atlantic Ocean, in Fig. 8 we have plotted the vertical phase of the jets determined from the complete set of ship-board profiles and from the mooring fits. To help judge any potential seasonal signal, the winter and summer seasons have been marked with different shading. The figure documents clearly that there is no seasonal signal, since a number of consecutive summers and winters have similar phase, while there are different summers that are approximately $180^{\circ}$ out of phase. Longer-term interannual variability is visible however, which from this sparse data looks somewhat periodic.

\section{Potential mechanism and effects}

In this section, the inertial instability theory, as first applied by Hua et al. (1996) to the equatorial stacked jets, is investigated as a possible explanation for this phenomenon. Other candidate mechanisms had been tested without success in previous studies (see introduction). The inertial instability theory requires an 

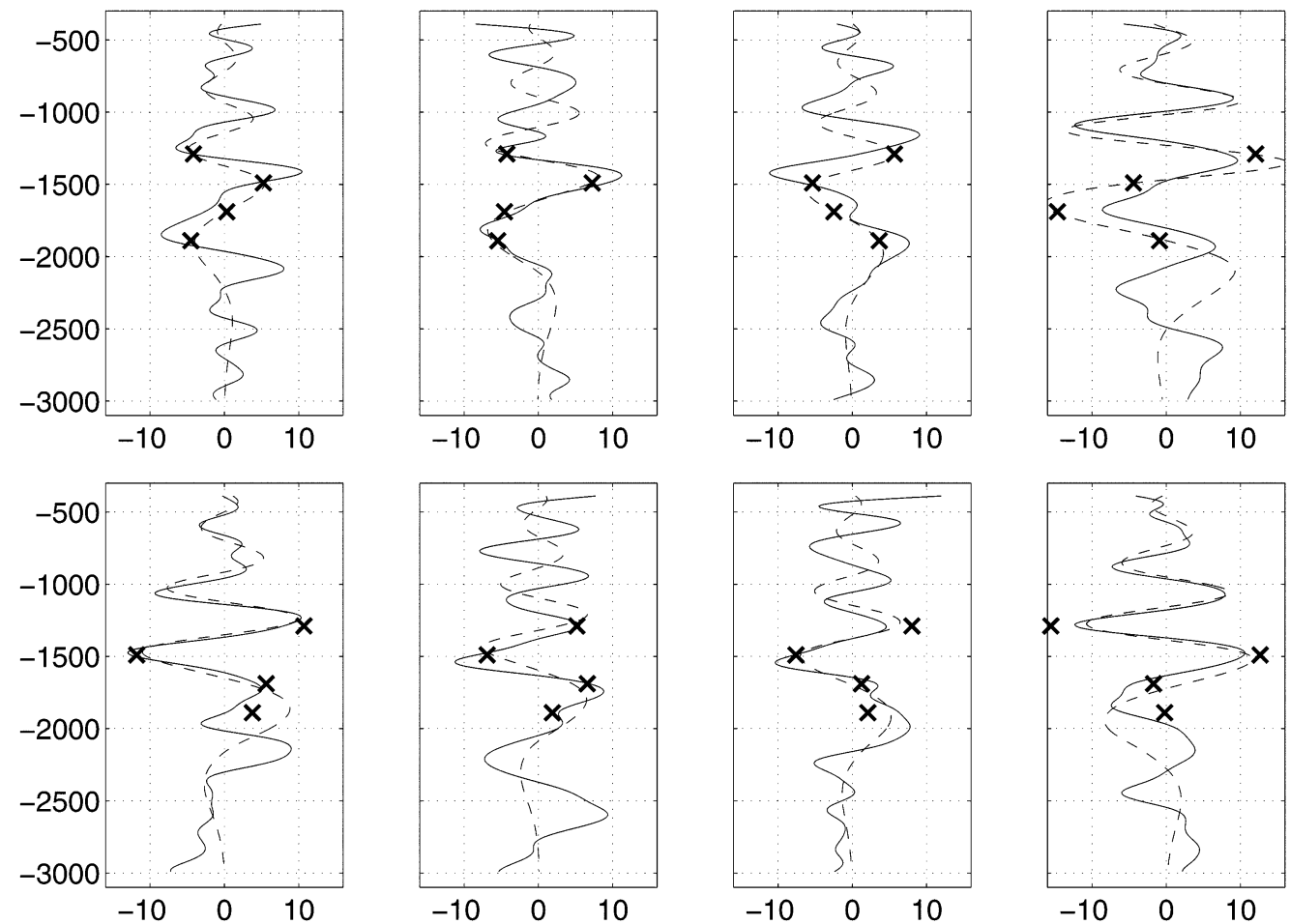

FIG. 6. Some examples of sampling an observed complete profile at four depths (1300, 1500, 1700, 1900 m) and fitting a jetlike structure from those four points (using a stretched wavelength of $550 \mathrm{~m}$ and a Gaussian tapering of $800 \mathrm{~m}$ ). The fits are the dashed lines, the solid lines denote the mode-filtered full profiles. The crosses denote the sampled currents of the complete profiles minus the estimated constant, which represents the background flow in the model. Such fits test the ability to use current meters at those depths for obtaining time series of the jets. Tests with 37 complete profiles from eight cruises gave an rms error in determining the vertical phase of the jets of $30^{\circ}$ and an rms misfit between fitted and complete profiles of $4 \mathrm{~cm} \mathrm{~s}^{-1}$.

asymmetry of the zonal flow about the equator and an associated meridional shear $\partial u / \partial y$ that is large enough to cancel the planetary vorticity $f$ (more rigorously, the Ertel potential vorticity has to vanish) as a necessary ingredient for the instability to develop. The instability takes the form of vertically stacked zonal jets trapped between the geographical and dynamical equator with very high vertical wavenumber. In linear theory this wavenumber is limited only by viscous effects, thus this parameter cannot be predicted by the theory at present. Attempts to date, however, consistently result in vertical scales that are significantly smaller than observed. For a given viscosity, the vertical wavelength scales approximately like $N^{-1}$. The solutions are stationary in space and induce a closed meridional circulation that acts to redistribute the asymmetric absolute angular momentum across the equator.

Hua et al. (1996) could only demonstrate that in the mean field containing the stacked jets, the zonal flow $u$ had a similar shape to $f y$ in the westward jets, thus there was a tendency for these terms to cancel. This was somewhat unsatisfactory, since the required shears in that case are a result of the instability, not a cause.

The eight sections we have available across the equator cannot be averaged as in Firing (1987) and
Hua et al. (1996) to enhance (in case of stationarity) or average out (in case of randomness) the jets. However, they can be used to highlight the large variability in the background flow from cruise to cruise. Figure 9 shows a rich structure of the zonal flows within a few degrees of the equator for each cruise, even after removing the jets by using only the lowest seven modes. As will be shown subsequently, the associated time-variable meridional shears can locally reach the same magnitude as $f$.

The necessary criterion for inertial instability of vanishing Ertel potential vorticity can be estimated for each realization from its dominant term $f-\partial u / \partial y$. Figure 10 shows the shape of this term as a function of latitude at two different depths. Despite the fact that the shears may be underestimated due to the discrete station sampling, repeatedly there are regions within $2^{\circ}$ of the equator where the potential vorticity vanishes. Inspection of the profiles shows that in the 1000-2000-m depth range the high shears mostly originate from anomalous (asymmetric) positive zonal momentum to the south of the equator. The observations thus suggest that the details of the instantaneous background flow may fulfil the instability criterion at variable locations in depth and $y$. 

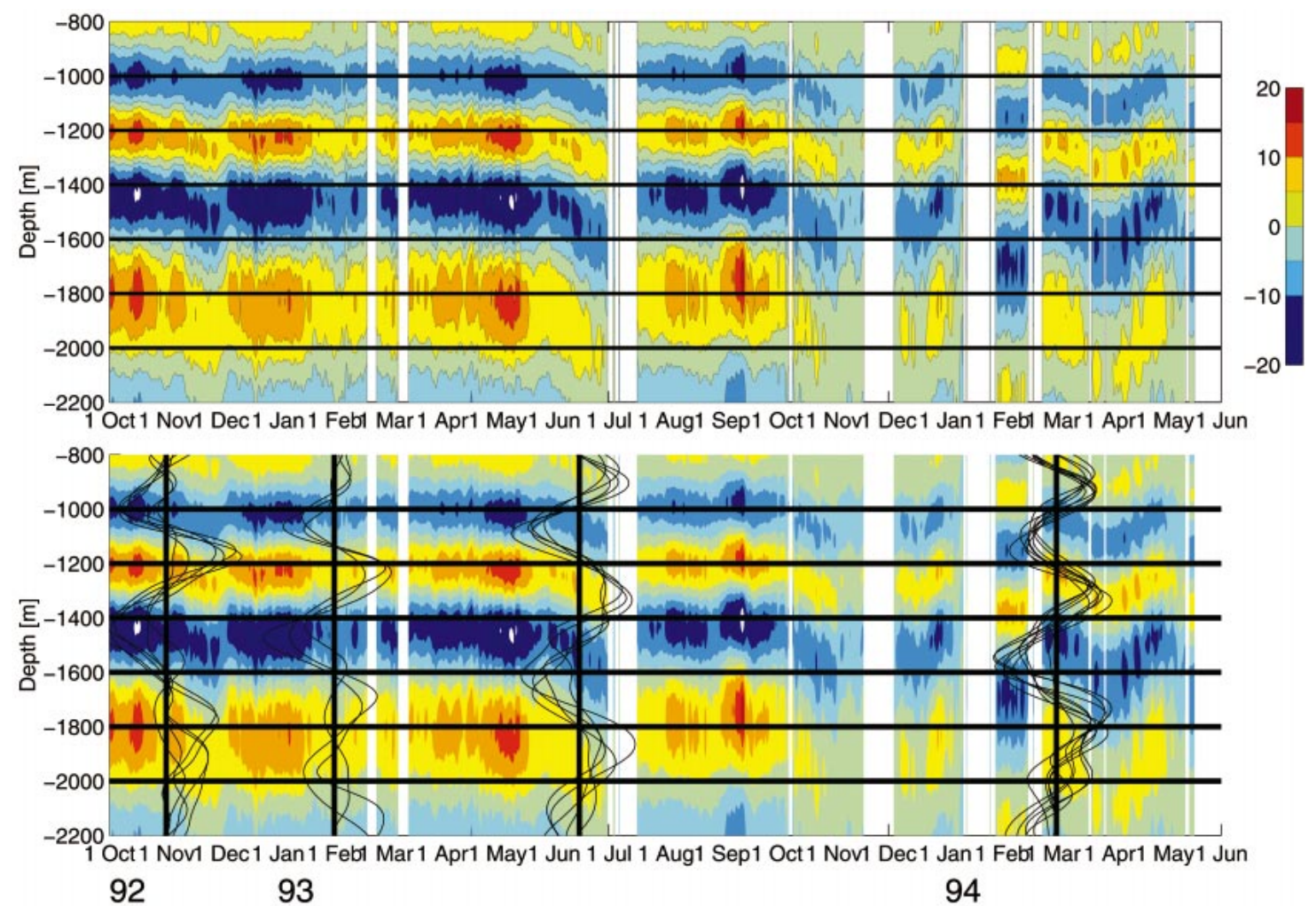

Fig. 7. (top) An estimate of the vertical jet structures using zonal velocity from four current meters on an equatorial mooring at $36^{\circ} \mathrm{W}$, according to the test performed in Fig. 6. The time series covers 20 months from fall 1992 until summer 1994. During this period, four ship-board sections were collected. (bottom). Their profiles are plotted for comparison at the corresponding times. Periods when the variance of all current meters was low are blanked out, since then the approach should be ill-determined. The estimated error in the vertical location of a jet is $50-70 \mathrm{~m}$ rms. For verification the lower panel shows full shipboard mode-filtered flow profiles at the times available, with a horizontal offset corresponding to the date of their observation (marked by the heavy line). It is underlaid with the same distribution as the upper panel for ease of comparison.

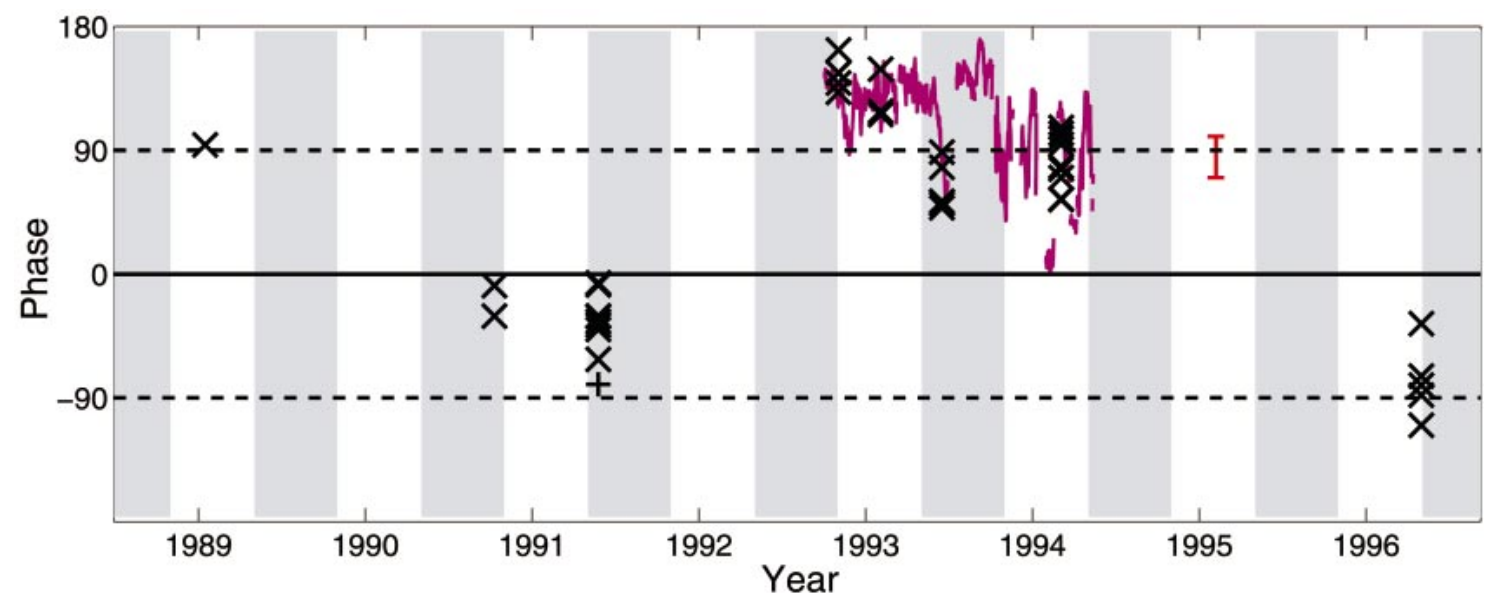

FIG. 8. Composite time series of all information available about the vertical phase of the jets, including published profiles. Vertical phase for ship-board profiles (crosses) was estimated by a 550-m wavelength fit equivalent to the treatment of the current meter time series, which is also included (magenta line). The different crosses grouped together at single dates correspond the the various profiles within $\pm 1^{\circ}$ of the equator. The error bar corresponds to $30^{\circ}$ uncertainty. Summer seasons are marked by gray shading to facilitate judgement of potential seasonal behavior. The agreement of ship observations with the current meter data is apparent again. Reversals of the jets (phase changes by $180^{\circ}$ ) are seen occasionally, but without connection to seasons. 

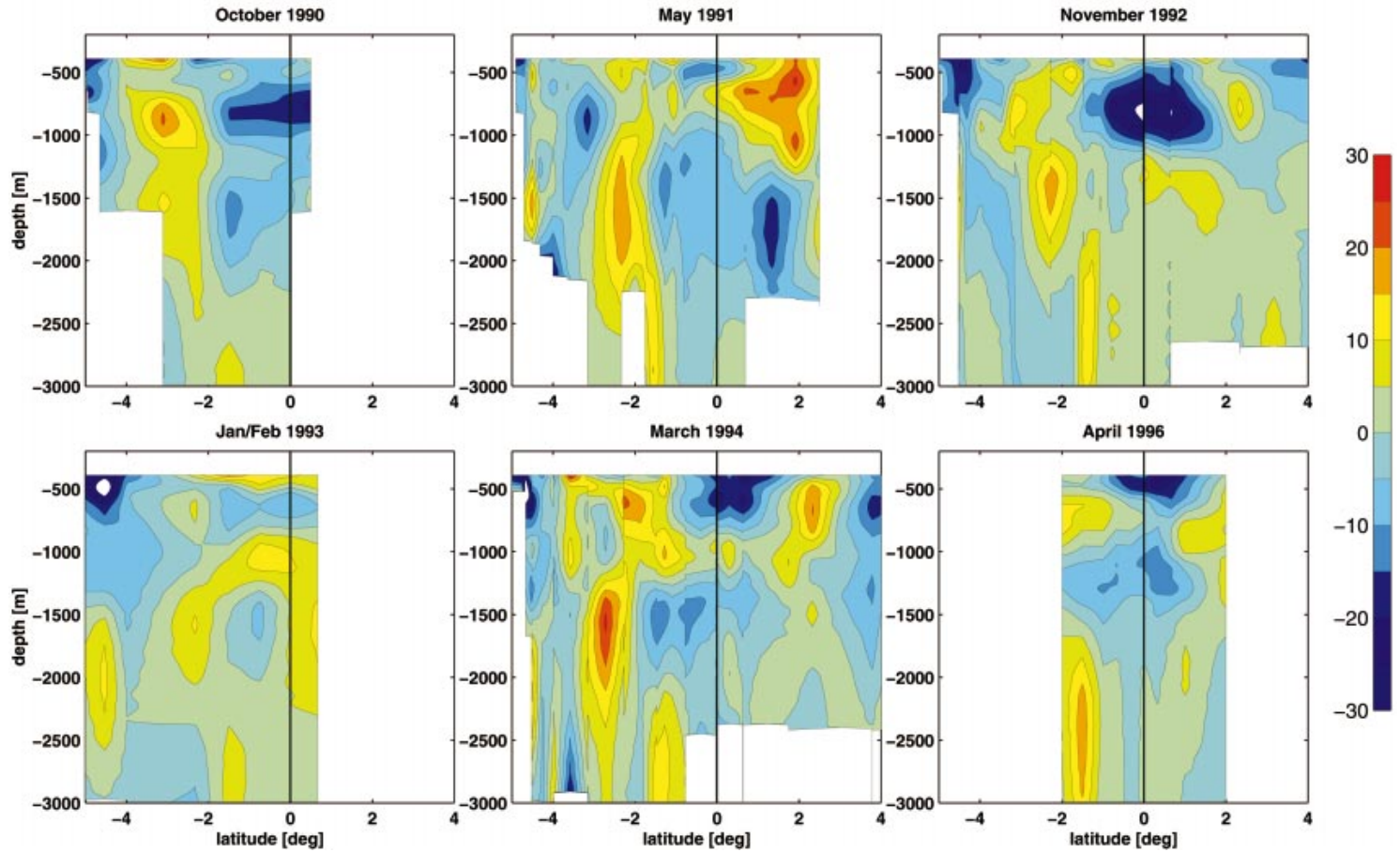

FIG. 9. Cross-equatorial sections of the background (low-mode) zonal flow from six cruises in $\mathrm{cm} \mathrm{s}^{-1}$. Pronounced asymmetries and flow cores with large shears are seen that change from cruise to cruise. Especially in the 1000-2000 m depth range there is frequently an excess of positive zonal (i.e., angular) momentum south of the equator in the complete sections.
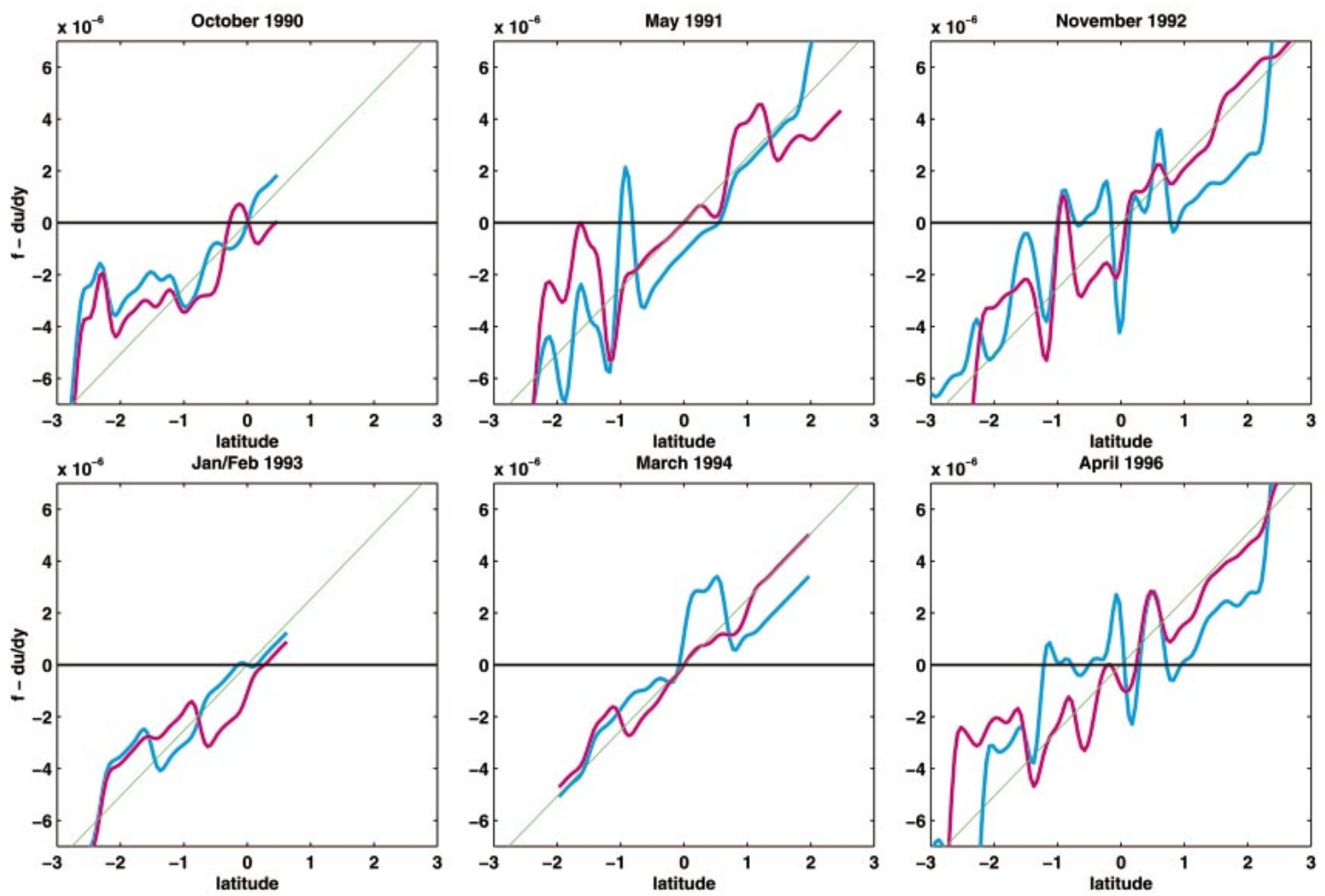

FIG. 10. The dominant term of the Ertel PV $(f-\partial u / \partial y)$ estimated for each section shown in the previous figure, at depths around 700 $\mathrm{m}$ (blue) and $1500 \mathrm{~m}$ (red). Note that the time-mean flow (not shown) is smoother and may not exhibit enough shear to overcome $f$ (green line). 


\section{Cross-equatorial angular momentum flux}

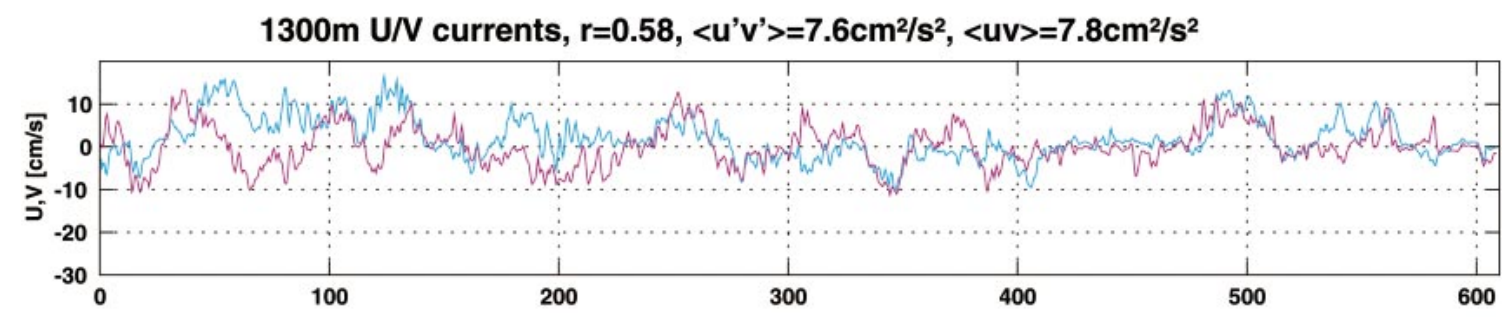

$1500 \mathrm{~m} U / V$ currents, $r=0.15,\left\langle u^{\prime} v^{\prime}\right\rangle=0.86 \mathrm{~cm}^{2} / \mathrm{s}^{2},\langle u v\rangle=0.08 \mathrm{~cm}^{2} / \mathrm{s}^{2}$

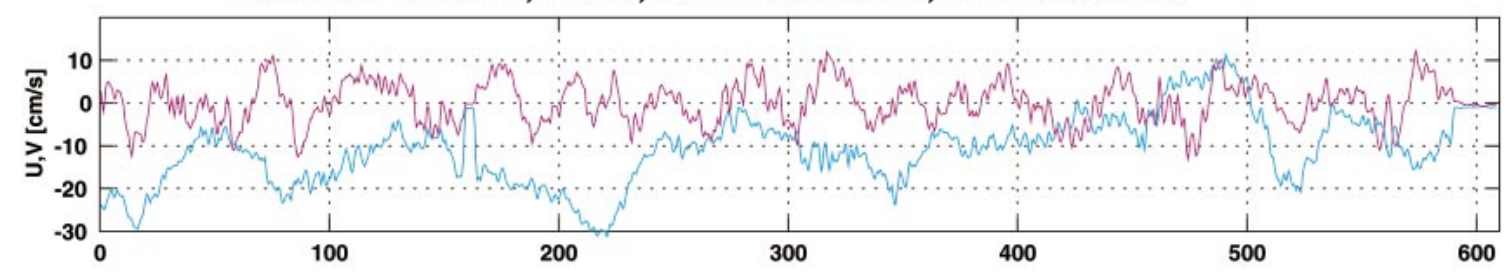

$1700 \mathrm{~m}$ U/V currents, $r=0.63,\left\langle u^{\prime} v^{\prime}\right\rangle=6.7 \mathrm{~cm}^{2} / \mathrm{s}^{2},\langle u v\rangle=6.4 \mathrm{~cm}^{2} / \mathrm{s}^{2}$

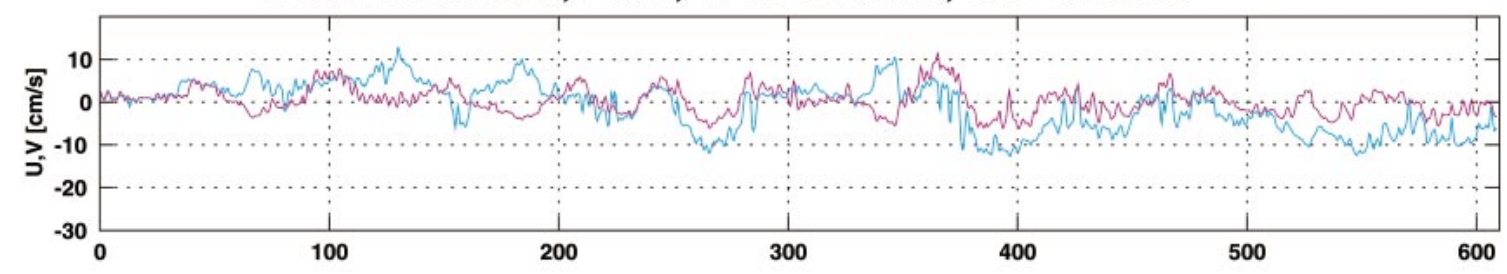

$1900 \mathrm{~m} \mathrm{U} / \mathrm{V}$ currents, $\mathrm{r}=0.58,\left\langle\mathrm{u}^{\prime} \mathrm{v}^{\prime}\right\rangle=9.5 \mathrm{~cm}^{2} / \mathrm{s}^{2},\langle u v\rangle=14.7 \mathrm{~cm}^{2} / \mathrm{s}^{2}$

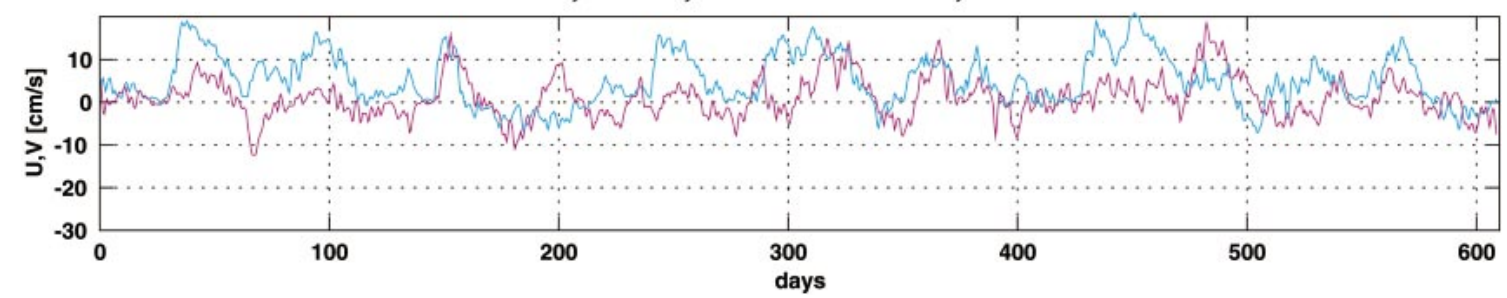

FIG. 11. Time series of $u$ (blue) and $v$ (red) from the four moored sensors at 1300-1900 m. The correlation coefficient, the covariance, and mean product are listed above the subpanels in each case. All sensors show a northward flux of momentum over the 20-month time period. The mean meridional flows $\langle v\rangle$ are small $\left(0.1,0.1,0.3\right.$, and $1.0 \mathrm{~cm} \mathrm{~s}^{-1}$ for the $1300-1900-\mathrm{m}$ levels, respectively).

We have no information on the persistance and timescale for these shears.

If inertial instability was the cause for the stacked jets and if it is related to the excess of zonal momentum on the southern side, one would expect a northward flux of zonal momentum associated with the stacked-jet flows, at least in the depth range where the meridional asymmetries are found. An attempt was made to estimate this flux at the location of the four current meters. Since we do not know how to identify the meridional flow $v$ that might be associated with a meridional circulation of the instability (in the presence of other flows), we can only calculate the full northward flux of zonal momentum $\langle u v\rangle$, where \langle\rangle is a time mean and $u, v$ are the raw (25-h lowpassed) zonal and meridional current meter time se- ries. For comparison the eddy fluxes were also estimated; that is, $\left\langle u^{\prime} v^{\prime}\right\rangle$, where the prime denotes the deviation from the time mean.

As Fig. 11 shows, three of the four current meters have appreciable northward flux of zonal momentum both in the time mean and in the fluctuating flow components. The correlations of those three over the 20month time series are 0.5-0.6 and the fluxes are in the range $6-14 \mathrm{~cm}^{2} \mathrm{~s}^{-2}$. Thus the northward momentum flux is in principle consistent with the expectation from the theory. However, the depth levels of the fluxes are in contradiction with, for example, H96, if the vanishing flux at the $1500-\mathrm{m}$ instrument is significant. This is the depth of a strong westward jet most of the time, and the theory predicts maximum meridional flux at these depths. 


\section{Summary and conclusions}

We have used a large number of shipboard flow profiles, covering eight periods over $7 \mathrm{yr}$, and 20-monthlong current meter time series, to analyze the meridional, depth, and time structure of deep zonal jets in the equatorial Atlantic. Comparing stretched vertical wavenumber spectra of the zonal flow on the equator with locations more than $1.5^{\circ}$ away from it, the jets were clearly visible as regions of elevated equatorial energy in a well-defined wavenumber band. Using this definition, mode filtering could be used to isolate the spatial variability associated with these features in individual current profile sections.

The vertical structure of the jets can be approximated by stretched sine/cosine functions with a Gaussian amplitude envelope, allowing $82 \%$ of the variance in all near-equatorial profiles to be explained. Using a fixed mean wavenumber and envelope length scale, the time series from four current meters could be used to construct a 20-month time series of the vertical phase of the jets. There are long periods of stationarity, alternating with intervals of more erratic behavior. Over the moored period, the phase did not change by more than $90^{\circ}$. Adding all the ship-board observations, a pronounced interannual variability is documented including reversals of the flow, but a seasonal signal is clearly not present.

Our analyses have found some features that are consistent with the inertial instability theory of Hua et al. (1996) as a possible mechanism for the generation of the jet structures. While the necessary condition for the instability is usually not satisfied by the mean flow conditions, it appears that the instantaneous meridional shears present in snapshots of the time-variable flow can bring the Ertel Potential vorticity (PV) to zero locally. Whether these shears persist sufficiently long to drive the instability remains an open question at the moment. A northward flux of zonal momentum, as one might expect from the theory in the case of an excess of angular momentum south of the equator, was diagnosed at three of the four current meters. However, the depth levels do not appear consistent with the theory, as do the relatively long vertical scales. A meridional offset of the jets (between geographical and dynamical equator) could not be diagnosed with our data, possibly due to insufficient meridional sampling.

The implications that the instability process would have are worth considering. The mechanisms for the exchange of water and water mass properties across the equator are a topic of ongoing discussion. Some of the deep water masses cross the equator as a deep boundary current, but also a branching of the flow along the equator is revealed by direct flow observations [e.g., float tracks, Richardson and Schmitz (1993)] and by salinity/tracer observations. Because of the strong zonality of the latter flows, this water can only be exchanged across the equator through an efficient mixing process. It could be then that the me- ridional transfers associated with the inertial instability process is the agent for this mixing. In this case, while the zonal flows are the most visible property of the deep jets, their effect on meridional exchanges is the more dynamically important factor. However, conclusive evidence for this role of deep jets is missing. We were unable to extract a meridional flow pattern that is spatially coherent with the zonal flow sections, possibly because the meridional flows are contaminated by higher frequency fluctuations. The four current meter time series also do not allow a sufficiently accurate extraction of the jet signals to compare their zonal and meridional flow components. A useful next step might consist of dedicated numerical simulations including the transient shears that fulfill the instability criterion in our observations, in order to test whether they can persist sufficiently long to trigger an instability.

Acknowledgments. Y. Gouriou, H. Mercier, and W. Johns made available the data from the Cither, Romanche, Etambot, and NOAA-AOML cruises for this study, which is gratefully appreciated. The success of the German Meteor cruises is due largely to the competence and cooperation of the master and the crew of the ship, as well as the technical staff who joined the cruise. We thank them for their support.

\section{REFERENCES}

Dunkerton, T. D., 1981: On the inertial stability of the equatorial middle atmosphere. J. Atmos. Sci., 38, 2354-2364.

, 1983: A nonsymmetric equatorial inertial instability. J. Atmos. Sci., 40, 807-813.

Eriksen, C. C., 1982: Geostrophic equatorial deep jets. J. Mar. Res., 40 (Suppl.), 143-157.

Firing, E., 1987: Deep zonal currents in the central equatorial pacific. J. Mar. Res., 45, 791-812.

Fischer, J., and M. Visbeck, 1993: Deep velocity profiling with selfcontained ADCP. J. Atmos. Oceanic Technol., 10, 764-773.

Gouriou, Y., B. Bourles, H. Mercier, and R. Chuchla, 1999: Deep jets in the equatorial Atlantic Ocean. J. Geophys. Res., 104, $21217-21226$.

Hayes, S. P., and H. B. Milburn, 1980: On the vertical structure of velocity in the eastern equatorial pacific. J. Phys. Oceanogr., 10, 633-635.

Hua, L. B., D. W. Moore, and S. Le Gentil, 1996: Inertial nonlinear equilibrium of equatorial flows. J. Fluid Mech., 320, 1-27.

Leetma, A., and P. F. Spain, 1981: Results from a velocity transect along the equator from $125^{\circ}$ to $159^{\circ}$ W. J. Phys. Oceanogr., 11, 1030-1033.

Lutyen, J. R., and J. C. Swallow, 1976: Equatorial undercurrents. Deep-Sea Res., 23, 1005-1007.

McCreary, J. P., 1984: Equatorial beams. J. Mar. Res., 42, 395-450.

—_, and R. Lukas, 1986: The response of the equatorial ocean to a moving wind field. J. Geophys. Res., 91, 11 691-11 705.

Muench, J., E. Kunze, and E. Firing, 1994: The potenial vorticity structure of equatorial deep jets. J. Phys. Oceanogr., 24, 418428.

Ponte, R. M., and J. Luyten, 1989: Analysis and interpretation of deep equatorial currents in the central pacific. J. Phys. Oceanogr., 19, 1025-1038. 
and $\longrightarrow$ 1990: Deep velocity measurements in the western equatorial Indian Ocean. J. Phys. Oceanogr., 20, 44-52.

Richardson, P. L., and W. J. Schmitz Jr., 1993: Deep cross-equatorial

flow in the Atlantic measured with sofar floats. J. Geophys. Res. 98, 8371-8387.

Spain, P. F., D. L. Dorson, and H. T. Rossby, 1981: Pegasus: A simple, acoustically tracked velocity profiler. Deep-Sea Res., 28A, 15531567.

Stevens, D. E., 1983: On symmetric stability and instability of zonal mean flows near the equator. J. Atmos. Sci., 40, 882-893.

Wunsch, C., 1977: Response of an equatorial ocean to a periodic monsoon. J. Phys. Oceanogr., 7, 497-511. 